\title{
Smoking cessation and lung cancer screening
}

\author{
Jesper Holst Pedersen ${ }^{1}$, Philip Tønnesen ${ }^{2}$, Haseem Ashraf ${ }^{2,3}$ \\ ${ }^{1}$ Department of Thoracic Surgery RT, Rigshospitalet, University of Copenhagen, Copenhagen, Denmark; ${ }^{2}$ Department of Respiratory Medicine, \\ Gentofte University Hospital, Hellerup, Denmark; ${ }^{3}$ Department of Radiology, Akershus University Hospital, Lørenskog, Norway \\ Contributions: (I) Conception and design: H Ashraf, P Tønnesen; (II) Administrative support: H Ashraf, JH Pedersen; (III) Provision of study \\ materials or patients: H Ashraf, P Tønnesen; (IV) Collection and assembly of data: H Ashraf, P Tønnesen; (V) Data analysis and interpretation: All \\ authors; (VI) Manuscript writing: All authors; (VII) Final approval of manuscript: All authors. \\ Correspondence to: Haseem Ashraf, MD, PhD. Chief Consultant, Head of Residency Program, Department of Radiology, Akershus University \\ Hospital, Norway. Email: haseem.ashraf@gmail.com.
}

\begin{abstract}
Smoking behavior may have a substantial influence on the overall effect of lung cancer screening. Non-randomized studies of smoking behavior during screening have indicated that computer tomography (CT) screening induces smoking cessation. Randomized studies have further elaborated that this effect has to do with participation in screening alone and not dependent on the CT scan. Participants in both CT and control arm in randomized screening trials had higher smoking abstinence rate compared to that of the general population. A positive screening test seems to further promote smoking cessation and decrease smoking relapse rate. Also low smoking dependency and high motivation to quit smoking at baseline predicted smoking abstinence in screening trials. Lung cancer screening therefore seems to be a teachable moment for smoking cessation. Targeted smoking cessation counselling should be an integrated part of future lung cancer screening trials.
\end{abstract}

Keywords: Lung cancer screening; smoking; smoking cessation; computer tomography (CT) scanning; positive findings; review

Submitted Jan 09, 2016. Accepted for publication Feb 05, 2016.

doi: $10.21037 /$ atm.2016.03.54

View this article at: http://dx.doi.org/10.21037/atm.2016.03.54

\section{Introduction}

Smoking is well known to be the main cause of lung cancer, which is the number one cause of cancer related death worldwide $(1,2)$. Lung cancer screening with low dose computer tomography (CT) can achieve early detection at a stage where curative treatment still is possible (3). The National Lung Screening Trial (NLST) with 53,452 participants screened current and former smokers with a smoking history of 30 pack years or more in USA (4). The NLST study is the first study that has demonstrated a significant $20 \%$ lung cancer mortality reduction and because of this national screening program is underway in the US (5) and is being considered in Europe (6). However, the overall success of lung cancer screening programs may be significantly influenced by any direct effect of screening on smoking behavior of the screened participants and therefore this should also be investigated.
This review focuses on smoking behavior and on predictors for smoking cessation in relation to lung cancer screening with low dose CT.

\section{License to smoke or teachable moment?}

One possible drawback of lung cancer screening may be that it can induce a false sense of safety and security in the participants so that they may feel protected against the harmful effect of smoking (7). The participants may therefore be demotivated to quit smoking or even worse relapse to smoking. Previous non-randomized studies have called this a "license to smoke" for the participants and is one of the main concerns against the implementation of lung cancer screening. A possible "license to smoke" effect may therefore jeopardize any possible beneficial mortality reduction effect of lung cancer screening (8).

On the other hand, some studies have suggested that 


\section{Page 2 of 5}

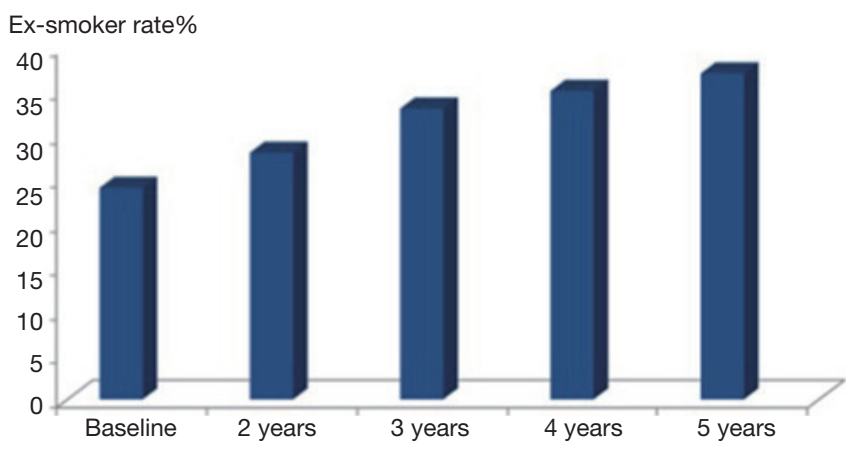

Figure 1 Development of ex-smokers rate in \% during the Danish Lung Cancer Screening Trail.

enrolment in screening may prove to be a teachable moment where the participants can be informed of the harmful effects of smoking and from this increase their motivation to quit smoking $(8,9)$.

To investigate the causal effect of lung cancer screening on smoking habits the best method is therefore to assess the smoking behavior in randomized settings.

\section{Randomized studies of smoking behavior in lung cancer screening}

Since the NLST study was published, a number of randomized European trials have also investigated the effect of lung cancer screening $(6,10,11)$. The aim of these studies was mainly to investigate if the mortality reduction from the NLST could be reproduced. Only two European studies also investigated the smoking habits of the participants enrolled in randomized screening trials. The Dutch-Belgian screening study (NELSON) (12) and the Danish Lung Cancer Screening Trial (DLCST) (13) have both published their analysis of the effect of screening on smoking habits. The NELSON study investigated a cohort of only male participants (12), and the DLCST is the only known study, which included all participants of both sex during all its five screening rounds (14). When analyzing smoking habits it is important to validate the smoking habits biochemically. In the DLCST this was done by analysis of carbon monoxid (CO) levels in exhaled breath. Subjects with a CO-level above $10 \mathrm{ppm}$ were regarded as current smokers (13).

DLCST was the first to publish its results regarding smoking habits after one year of screening in 2012 (13). The DLCST participants were randomized into two groups, one that was screened with low dose CT (screening group, $n=2,052$ ) and one group receiving no screening (control group, $\mathrm{n}=2,052)$. In both groups brief smoking cessation counseling was given by trained nurses but no active pharmacological or other smoking intervention was done. Thus, this design allowed a direct evaluation of the effect of CT screening on smoking habits by comparison between the two groups. The DLCST found no significant overall difference in smoking quit rates $(\mathrm{P}=0.49)$ between the screening group $[11 \%(174 / 1,545)]$ and control group [10\% $(165 / 1,579)]$ after 1 year of screening. However, as $17 \%(85 / 507)$ of the ex-smokers in the CT-group and $21 \%(98 / 473)(\mathrm{P}=0.11)$ of the ex-smokers in the control group relapsed to smoking, the net smoking quit rate was $6 \%$ (13). Thus, CT-screening by itself did not increase quitting compared to the control group. That $11-12 \%$ in both groups did quit smoking could be an effect of the brief smoking cessation counseling, combined with the fact that this group of smokers volunteering for a CT screening trial might be more motivated to quit than the background population.

The NELSON study reported quit rates in the same range as from the DLCST, i.e., a quit rate of 13\% (84/641) in the CT-group smokers versus $15 \%(96 / 643)(\mathrm{P}=0.3)$ in the control group smokers after approx. 2 years (8). However, they did not report number of relapsers in ex-smokers.

Later in 2014 the DLCST published its analysis of all its five screening rounds (14), and the conclusion was the same, that no significant difference in smoking behavior was seen between the screening and control group. CTscreening did not induce smoking nor did it promote smoking cessation when compared to the control group. But overall in both CT and control group ex-smoker rate increased from $24 \%$ at baseline to $37 \%$ at fifth screening round after 4 years [Figure 1, (14)]. Apparently, participation in a screening trial by itself promoted smoking cessation in both groups. One main problem that may introduce bias in smoking habits analyses is missing values concerning smoking status mainly in subject's lostto follow-up. Normally like in the DLCST $(13,14)$ such subjects should be regarded as current smokers in the analyses as most of these subjects are current smokers.

\section{Non-randomised trials}

Only 3 cohort studies—all conducted in US-reported smoking habits especially in relation to positive or negative findings on CT-scans. These studies are systematically reviewed recently (15). In the Mayo study with 1,475 smokers and ex-smokers the 1 -year quit rate was $12.1 \%$ 
$(127 / 1,051)$ and the relapse rate was $6.6 \%(58 / 874)$ in exsmokers (16). In the PLuSS study with 3,642 smokers and ex-smokers the 1 -year quit rate was $15.5 \%(325 / 2,094)$ with no data about relapsers (15). The last study_ELCAP—was a report in a low number of smokers that found a very high quit rate of $40 \%(66 / 134)$ an unspecified amount of time after CT-screening, and thus the results regarding quit rates is probably not valid (15).

Data regarding smoking has recently been published from a sub-population of the NLST, i.e., in 18,840 subjects out of 53,452 participants (15). Although this is a randomized controlled trial with ordinary chest $\mathrm{X}$-ray in the control group, they did not report smoking data for the control $\mathrm{arm}$. The annual point prevalence quit rate during 5 years ranged from $11.6 \%$ to $13.4 \%$.

Overall, the above 3 large cohort studies reported change in smoking prevalence in the same range as in the 2 randomized, controlled trials [DLCST $(13,14)$ and Nelson $(8)$ ]. It's important to underline that this decline in smoking rate most probably is not due to the CT-screening per se but instead an overall effect of participation in screening trials, and the selection bias induced by comparing quit rates in a highly motivated screened population with quit rates in the background population.

\section{Predictors for smoking abstinence in lung cancer screening trials}

One might suspect that there could be a differential effect from a negative (i.e., normal) or a positive CT-scan result on smoking habits. Positive CT-scan results might "scare" subjects and get them to quit smoking and decrease relapse in ex-smokers. Here randomized controlled trials and cohort studies may be of similar validity.

In the NELSON trial, a significant higher number of quit attempts was reported in participants receiving a positive screening result (13).

In the DLCST, a positive screening result significantly increased smoking cessation rate and decreased smoking relapse rate (13). However, overall during all five screening rounds in DLCST smoking behavior in those with positive screening results did not differ significantly from those with a negative screening result (14). This could suggest that the smoking cessation effect of a positive result might be shortlived and therefore intensive smoking cessation counseling in the immediate time after a positive screening test result could help further boost smoking abstinence.

These findings are also consistent with the analysis of smoking habits in the large NLST study cohort of smokers and found reduced smoking rate in participants with positive screen result. NLST results also showed a strong doseresponse in increased smoking cessation as screening results became more serious or suspicious for lung cancer (17). In the NLST study the first positive scan increased abstinence significantly with a HR of 1.28 (95\% CI, 1.13-1.35) and with a 1 -year abstinence rate of $15.9 \%$ ( $\mathrm{n}=1,869$ quitters) vs. $12.7 \%(\mathrm{n}=723$ quitters) after a second positive scan $(\mathrm{P}=0.04)$. They name the positive scans "false positive scans" as all the lung cancer cases were excluded from the analysis (13). For recent ex-smokers (or recent quitters), i.e., subjects that quit smoking within the last 6 months a positive scan was associated with less likelihood of relapse with a HR of 0.72 (95\% CI, 0.54-0.94). The proportion of recent quitters that relapsed the year following a positive screen was $40.8 \%(\mathrm{n}=120)$ compared with $10.8 \%(\mathrm{n}=37)$ in subjects with a second positive scan $(\mathrm{P}<0.001)$. Among longer-term quitters there was no relation with positive screen results and relapse $(16,17)$.

In summary, the NLST findings support that "false" positive screening test result increased smoking cessation and decreased relapse in recent quitters. Recurrent negative screens did not promote relapse in long-term quitters.

In the DLCST Fagerstroem first Questionnaire Q1 ("How soon after you wake up do you smoke your first cigarette?") was used to evaluate smokers' nicotine dependency (High dependency = early first cigarette) (13). Lower Fagerstroem dependency and higher motivation to quit at baseline predicted smoking status both after 1 year of screening and at the end of 5 -year screening program $(13,14)$. In the NLST study $74 \%$ of the smokers reported one or more quit attempts during the study period underlining that the majority of participants in these programs in fact try to quit smoking. A genetic study, which also included DLCST participants, found gene sequence variants associating with smoking behavior and with nicotine metabolizing enzymes (18). This may open for future genetic profiling of smoking behavior and from this design targeted smoking cessation intervention. Targeted smoking cessation of screening participants with high dependency could be a beneficial approach. This could be done by using pharmaceutical smoking cessation drugs combined with counseling for high dependent participants and a motivational approach for those subjects low in motivation to quit (19).

Recent data from a subgroup of the NLST in 3,336 quitters and continued smokers (20) reported that primary care providers with respect to smoking cessation support 
only partially followed the 5 As recommendations [i.e., ask about smoking (77\%), advise to quit (76\%), assess smoking status (63\%), assist (56\%) and arrange follow-up (10\%)]. There was no effect on smoking abstinence from "ask", "advise" and "assess" but "assist" increased odds of quitting with $40 \%$ and "arrange follow-up" increased odds of quitting with $46 \%$ (16). Thus, clinicians should intervene more actively in smoking cessation in the future screening trials especially with focus on assisting smokers to quit (talking about quitting and recommend smoking cessation drugs and/or counseling) and arrange follow-ups.

\section{Conclusions}

Overall, CT screening for lung cancer in itself does not result in smoking cessation (14). However, apparently participants in these programs are more motivated to quit smoking than the general background population, resulting in an overall quit smoking rate of approx. $10-13 \%$ during 4-5 years unrelated to the exposure to CT scanning $(13,14)$. There is some evidence that positive CT screening test results are associated with short-lived increases in quitting smoking and in reducing relapse in recent quitters with no consistent long-term effects $(13,14)$.

The success of future lung cancer screening programs may very well be dependent on the ability of promoting smoking cessation. Randomized trials that have investigated smoking behavior have shown that screening is a teachable moment for smoking cessation. The DLCST found overall increasing smoking cessation rate through its five screenings round. However, the smoking behavior of volunteers in screening trials may differ from those in the general population. Participation bias is well known from other screening studies, and this was also seen in the DLCST in which participants in both screening and control group were from higher social class compared to general smokers (21). The general population of smokers may have a lower motivation for smoking cessation. By assessing nicotine dependency and motivation for smoking cessation, targeted counseling could help further promote smoking abstinence in the general smoking population.

A comprehensive smoking cessation program should therefore be an integrated part of future screening programs. Despite the success of screening for lung cancer with low dose CT, smoking cessation still is the most effective tool in the battle against not only lung cancer but also cardiovascular disease and COPD. A successful and targeted smoking cessation counseling programs in lung screening programs may therefore be the most effective method to reduce mortality of thoracic smoke related diseases.

\section{Acknowledgements}

None.

\section{Footnote}

Conflicts of Interest: The authors have no conflicts of interest to declare.

\section{References}

1. Sasco AJ, Secretan MB, Straif K. Tobacco smoking and cancer: a brief review of recent epidemiological evidence. Lung Cancer 2004;45 Suppl 2:S3-9.

2. Loeb LA, Ernster VL, Warner KE, et al. Smoking and lung cancer: an overview. Cancer Res 1984;44:5940-58.

3. van Klaveren RJ, Habbema JD, Pedersen JH, et al. Lung cancer screening by low-dose spiral computed tomography. Eur Respir J 2001;18:857-66.

4. National Lung Screening Trial Research Team, Aberle DR, Adams AM, et al. Reduced lung-cancer mortality with low-dose computed tomographic screening. $\mathrm{N}$ Engl J Med 2011;365:395-409.

5. Fintelmann FJ, Bernheim A, Digumarthy SR, et al. The 10 pillars of lung cancer screening: rationale and logistics of a lung cancer screening program. Radiographics 2015;35:1893-908.

6. Saghir Z, Dirksen A, Ashraf H, et al. CT screening for lung cancer brings forward early disease. The randomised Danish Lung Cancer Screening Trial: status after five annual screening rounds with low-dose CT. Thorax 2012;67:296-301.

7. Taylor KL, Cox LS, Zincke N, et al. Lung cancer screening as a teachable moment for smoking cessation. Lung Cancer 2007;56:125-34.

8. van der Aalst CM, van den Bergh KA, Willemsen MC, et al. Lung cancer screening and smoking abstinence: 2 year follow-up data from the Dutch-Belgian randomised controlled lung cancer screening trial. Thorax 2010;65:600-5.

9. Bach PB, Mirkin JN, Oliver TK, et al. Benefits and harms of CT screening for lung cancer: a systematic review. JAMA 2012;307:2418-29.

10. Xu DM, Gietema H, de Koning H, et al. Nodule management protocol of the NELSON randomised lung 
cancer screening trial. Lung Cancer 2006;54:177-84.

11. Infante $M$, Cavuto S, Lutman FR, et al. A randomized study of lung cancer screening with spiral computed tomography: three-year results from the DANTE trial. Am J Respir Crit Care Med 2009;180:445-53.

12. van der Aalst CM, van Klaveren RJ, van den Bergh KA, et al. The impact of a lung cancer computed tomography screening result on smoking abstinence. Eur Respir J 2011;37:1466-73.

13. Ashraf H, Tønnesen P, Pedersen JH, et al. Smoking habits were unaffected by CT screening at 1-year follow-up in the Danish Lung Cancer Screening Trial (DLCST). Thorax 2009;64:371-2.

14. Ashraf H, Saghir Z, Dirksen A, et al. Smoking habits in the randomised Danish Lung Cancer Screening Trial with low-dose CT: final results after a 5 -year screening programme. Thorax 2014;69:574-9.

15. Slatore CG, Baumann C, Pappas M, et al. Smoking behaviors among patients receiving computed tomography for lung cancer screening. Systematic review in support of the U.S. preventive services task force. Ann Am Thorac Soc 2014;11:619-27.

Cite this article as: Pedersen JH, Tønnesen P, Ashraf H. Smoking cessation and lung cancer screening. Ann Transl Med 2016;4(8):157. doi: 10.21037/atm.2016.03.54
16. Clark MA, Gorelick JJ, Sicks JD, et al. The relations between false positive and negative screens and smoking cessation and relapse in the national lung screening trial: implications for public health. Nicotine Tob Res 2016;18:17-24.

17. Tammemägi MC, Berg CD, Riley TL, et al. Impact of lung cancer screening results on smoking cessation. J Natl Cancer Inst 2014;106:dju084.

18. Thorgeirsson TE, Gudbjartsson DF, Surakka I, et al. Sequence variants at CHRNB3-CHRNA6 and CYP2A6 affect smoking behavior. Nat Genet 2010;42:448-53.

19. Jiang B, He Y, Zuo F, et al. Effectiveness of varenicline and counselling for smoking cessation in an observational cohort study in China. BMJ Open 2016;6:e009381.

20. Park ER, Gareen IF, Japuntich S, et al. Primary care provider-delivered smoking cessation interventions and smoking cessation among participants in the national lung screening trial. JAMA Intern Med. 2015;175:1509-16.

21. Hestbech MS, Siersma V, Dirksen A, et al. Participation bias in a randomised trial of screening for lung cancer. Lung Cancer 2011;73:325-31. 